\title{
How Word-Of-Mouth Transmission Encouragement Affects Consumers' Transmission Decisions, Receiver Selection, and Diffusion Speed
}

\author{
Andrew T. Stephen \\ L'Oréal Professor of Marketing \\ University of Oxford, Saïd Business School \\ Park End Street, Oxford OX1 1HP, UK \\ Email. Andrew.Stephen@sbs.ox.ac.uk
}

Donald R. Lehmann

George E. Warren Professor of Business

Columbia University, Graduate School of Business

3022 Broadway, 507 Uris Hall, New York NY 10027, USA

Email.dr12@columbia.edu

Forthcoming, International Journal of Research in Marketing

Andrew T. Stephen (Andrew.Stephen@sbs.ox.ac.uk) is L'Oréal Professor of Marketing at the Saïd Business School, University of Oxford; Donald R. Lehmann (drl2@ columbia.edu) is George E. Warren Professor of Business at the Graduate School of Business, Columbia University. The authors thank Jonah Berger, Yaniv Dover, Jeff Inman, Kamel Jedidi, Leonard Lee, Jeff Parker, Olivier Toubia, Christophe Van den Bulte, Duncan Watts, Peter Zubcsek, and seminar participants at the Interdisciplinary Center, Herzliya, Israel for their comments and assistance with this research. This research was supported by the Katz Fellowship at the University of Pittsburgh during the time that the first author was a faculty member at that institution, and the L'Oréal Chair Fund at the Saïd Business School, University of Oxford. 


\title{
How Word-Of-Mouth Transmission Encouragement Affects Consumers' Transmission Decisions, Receiver Selection, and Diffusion Speed
}

\begin{abstract}
This research considers how marketers can encourage or "nudge" consumers to transmit word of mouth (WOM), such as referrals or recommendations to friends, in a manner that helps reach, inform, or influence large numbers of consumers quickly, which is an outcome referred to as faster diffusion. Building on studies showing diffusion is faster when higher-connectivity people are involved, the authors propose a mechanism based on network externalities that encourages regular customers to select receivers who have higher levels of social connectivity. Three experiments and a simulation demonstrate the mechanism's efficacy by showing (i) how regular consumers can be encouraged to select higher-connectivity friends as WOM receivers, (ii) why this mechanism works, and (iii) how encouraging this receiver selection behavior can lead to faster diffusion at the macro level. The findings extend the WOM marketing literature by introducing an approach for encouraging transmissions to specific types of consumers that is a viable alternative to directly targeting higher-connectivity consumers in seeded WOM campaigns, which is often practically infeasible.
\end{abstract}




\section{Introduction}

A common marketing objective is to have consumers transmit firm-related information, such as referrals and recommendations, to friends via word of mouth (WOM). The importance of WOM-based information sharing and referrals is well established in marketing (e.g., Godes and Mayzlin 2004, 2009; Libai et al. 2010; for reviews see Berger 2014, Lamberton and Stephen 2016, Stephen 2016), and WOM is a key driver of customer value (Kumar, Petersen, and Leone 2007; Kumar et al. 2010; Schmitt, Skiera, and Van den Bulte 2011). Getting people to spread awareness and information to friends via online and offline social networks in order to generate collective action is also important outside of marketing, where it is referred to as social mobilization (Pickard et al. 2011; Rutherford et al. 2013a, 2013b). A defining feature of social mobilization is faster information diffusion; i.e., information should reach as many people as possible in as little time as possible (Pickard et al. 2011). ${ }^{1}$ In WOM marketing, this implies that information spreads not only widely but also quickly. This is practically important in many situations, e.g., when racing against competition to attract new customers, when a promotion is available for a limited time, when managing a crisis (e.g., a recall), or when a new product has a short time to turn a profit (e.g., theatrical movie releases).

How can a WOM-driven, social mobilization outcome, characterized by faster diffusion, be encouraged by marketers when seeding WOM campaigns? Extant literature has considered how to encourage widespread diffusion. However, the rate at which this occurs and, specifically, faster diffusion, has received scant attention. An exception is Goldenberg, Han, Lehmann, and Hong (2009) who consider acceleration of diffusion processes in the context of virtual goods in an online social network, although the their focus is on macro-level outcomes instead of the

\footnotetext{
${ }^{1}$ Diffusion speed can be considered in two ways. First, the amount of time taken for information to spread to a fixed number of people (i.e., less time to reach a fixed number of people $=$ faster diffusion). Second, the number of people information spreads to in a fixed time period (i.e., more people in a fixed time $=$ faster diffusion).
} 
micro-level WOM transmission behaviors that affect such outcomes. Some prior work has taken a micro-level perspective (e.g., Berger 2011; Barasch and Berger 2014; Chen and Berger 2013; Frenzen and Nakamoto 1993; Ryu and Feick 2007), and of the research that focuses on microlevel aspects of WOM transmission that make macro-level diffusion outcomes more likely, two main approaches have emerged. One focuses on transmitter characteristics, and suggests that individual characteristics might make some people better WOM transmitters than others; e.g., opinion leadership and or market mavenism (e.g., Feick and Price 1987; Iyengar, Van den Bulte, and Valente 2011), customer loyalty (Godes and Mayzlin 2009), or customer value (Haenlein and Libai 2013). Note, however, that some doubts have been raised about the efficacy of socalled opinion leaders of "influentials" in driving diffusion (Iyengar et al. 2011). The other focuses on network characteristics, and suggests that some people are better WOM transmitters because they have larger social networks or higher social connectivity (i.e., "high degree" people or "hubs"), affording higher potential reach when they transmit (e.g., Goldenberg et al. 2009; Hinz, Skiera, Barrot, and Becker 2011). ${ }^{2}$

Both approaches emphasize transmitter-level characteristics that indicate a person is "special" and thus likely more effective as a WOM transmitter. Accordingly, research on seeded WOM marketing campaigns_—where firms solicit specific consumers as transmitters_-suggests that transmitter characteristics such as high connectivity are important when firms select seeds (e.g., Hinz et al. 2011). However, while it is logical to target consumers with special characteristics to be WOM transmitters, it is unfortunately often infeasible for marketers to identify, let alone recruit and activate, such individuals.

\footnotetext{
${ }^{2}$ Although high-connectivity hubs are seen as important drivers of diffusion, Watts and Dodds (2007) question their role and suggest instead that collective action depends on the susceptibility to influence of receivers.
} 
We propose an alternative approach for seeding WOM marketing campaigns that focuses specifically on faster diffusion as the desired macro-level goal (as opposed to diffusion without explicit concern for time). Contrary to prior research suggesting the direct targeting of special consumers as transmitters, we advocate the use of regular consumers-people who do not have special traits such as opinion leadership or high levels of social connectivity. Instead of focusing seeding on special transmitter characteristics, we consider how marketers can encourage or "nudge" regular consumers to direct their WOM transmissions to receivers who possess characteristics that will likely help accelerate, and thus quickly propel diffusion. Our approach shifts the focus from transmitter selection ("Who is the transmitter?") to receiver selection ("To whom is information transmitted?"), a topic which has been rarely addressed in research on firminitiated WOM marketing (a noteworthy exception is Frenzen and Nakamoto 1993). In line with prior work showing that diffusion accelerates once higher-connectivity people are reached and that social mobilization requires the involvement of higher-connectivity people (Goldenberg et al. 2009; Rutherford et al. 2013a), we consider how to encourage regular consumers to transmit WOM to their higher-connectivity friends.

This paper reports three experiments and a simulation showing (i) how regular consumers can be encouraged to select higher-connectivity friends as WOM receivers, (ii) why our mechanism, based on network externality, works, and (iii) that encouraging this receiver selection behavior can lead to faster diffusion at the macro level. Theoretically, we build on research on WOM referral incentives (e.g., Biyalogorsky, Gerstner, and Libai 2001; Ryu and Feick 2007; Schmitt et al. 2011; Verlegh, Ryu, Tuk, and Feick 2013) and social mobilization mechanisms (e.g., Pickard 2011; Rutherford 2013a, 2013b). Although incentives can be effective for increasing WOM transmission in general (e.g., Ryu and Feick 2007), prior research has not 
considered how marketers can influence receiver selection. Thus, our contribution lies in demonstrating how mechanisms can be used as "nudges" that affect transmitters' receiver selections in specific ways that can help marketers achieve their goals.

\section{Background and Conceptual Framework}

\subsection{Prior Literature}

As already noted, the current research is related to a number of research streams in marketing, as well as work on the role of incentives in large-scale social mobilization efforts in non-marketing contexts (Pickard 2011; Rutherford et al. 2013a, 2014b). A large body of research considers how marketers can encourage customers to talk about their products and services with their friends, and the positive effects WOM can have on firm outcomes such as customer acquisition, retention, and sales (e.g., Chevalier and Mayzlin 2006; Godes and Mayzlin 2004; Goldenberg, Libai, and Muller 2001, 2004; Stephen and Galak 2012; Trusov, Bucklin, and Pauwels 2009). The literature on customer value (e.g., Kumar et al. 2007; Kumar et al. 2010) also considers WOM in terms of customer-to-customer referrals, demonstrating that an important part of customer value comes from WOM referrals (see also Schmitt et al. 2011).

Research on WOM referral incentives or rewards is closely related to the current work. Studies on referral incentives consider factors such as the design of optimal reward programs to encourage customers to generate referrals (Biyalogorsky et al. 2001), when referral rewards increase existing customers' referral transmission likelihoods, and for what types of brands they are best suited (Ryu and Feick 2007). Other research considers receiver's reactions to WOM. Verlegh et al. (2013) show that receivers can react adversely because of suspicions of incentivized referrers having ulterior motives. Finally, Schmitt et al. (2011) find that referred 
customers are more valuable over time (e.g., less churn, more spending). Interestingly, this effect could be due to existing customers having superior knowledge of friends' interests and needs (compared to the firm), and therefore being able to find friends to refer to a firm who are a better match for the firm's offerings. More generally, this suggests it would be advantageous for firms to leverage customers' knowledge of friends' characteristics such as social connectivity.

\subsection{Mechanism for Influencing Transmission to Higher-Connectivity Friends}

We propose a mechanism for increasing the likelihood that WOM-transmitting customers select higher-connectivity friends as receivers. Incentives or rewards for transmitting WOM have been shown to work well in marketing settings in general (Ryu and Feick 2007), and the design of a promotion can influence transmitters' receiver choices (Frenzen and Nakamoto 1993). The social mobilization literature considers mechanisms designed to solve particular types of problems. For example, to win a contest in which teams had to find ten red weather balloons that the U.S. Defense Advanced Research Projects Agency placed at random locations, Pickard et al. (2011) implemented a "recursive" incentive mechanism that mobilized large numbers of people to search local areas. ${ }^{3}$ Pickard et al. (2011) argue that appropriate incentives are a key ingredient for successful social mobilization. Other studies in contexts such as collaborative problem solving and crowdsourcing also underscore the importance of incentives for regulating flows of information among socially connected actors in a manner that fosters desirable outcomes (e.g., Dodds, Muhamad, and Watts 2003; Mason and Watts 2009). In our context, a mechanism will be effective if it encourages transmitters to select higher-connectivity friends as receivers.

\footnotetext{
${ }^{3}$ See Pickard et al. (2011) for full details. Interestingly, their recursive incentive mechanism shared some similarities with "pyramid" incentive mechanisms that typically are illegal in person-to-person or "network" selling programs.
} 
To increase the probability of higher-connectivity receivers being selected, the social connectivity of one's potential receivers must be both considered by transmitters and deemed to be important. In other words, transmitters need to put some weight on receiver connectivity when deciding with whom they will share information. One way to encourage this to happen is to use an incentive mechanism that makes a transmitter's utility from sharing information with friends positively associated with the social connectivity of the transmitted-to friends. This will not happen naturally; it is unlikely that a regular consumer - as WOM transmitter-will put weight on the social connectivity of potential receivers without some inducement. This was shown to be the case in a pretest in which 448 U.S. members of Amazon Mechanical Turk were surveyed about WOM transmission. Respondents were asked to list receiver characteristics that they consider important when they decide with whom to share information via WOM. Only $3.8 \%$ of respondents mentioned something related to social connectivity (see Web Appendix A).

Given that transmitters do not typically consider receiver connectivity, how can marketers increase preference for higher-connectivity receivers? We posit that this can be achieved by satisfying two conditions. First, the macro-level faster-diffusion outcome must be important to transmitters. Second, transmitters must feel a sense of urgency with respect to deriving utility from their WOM transmission. Taken together, this means that, for example, transmitters should personally want many others to know about a talked-about product in as little time as possible, and feel some time pressure.

\subsection{Making Faster-Diffusion Outcomes More Important to Transmitters}

For receiver connectivity to positively influence a transmitter's receiver choice, a transmitter's individual, micro-level transmission utility needs to be positively associated with 
the macro-level firm-desired faster-diffusion outcome. In other words, transmitters need to care about faster diffusion (or social mobilization) and associate this with higher-connectivity receivers. If people possess an intuitive lay belief that diffusion will speed up when higherconnectivity people are involved, then they will have a preference for higher-connectivity receivers when they want the information they are transmitting to spread quickly.

Evidence of the prevalence of this lay belief was found in a pretest. We surveyed 100 U.S. members of Amazon Mechanical Turk and asked them to consider two scenarios. For the first scenario, they imagined that a friend posted a video on Facebook. We then asked them to select from a list of factors, including how funny the video was and, critically, the transmitter's level of social connectivity (number of friends), which ones they thought would affect how quickly the video would spread. Eighty-seven percent of respondents said that the video would spread faster if it was shared with someone who has many friends, i.e., higher connectivity.

For the second scenario, respondents imagined four new restaurants opening in their city and four different people transmitting WOM about each of these restaurants. The transmitters' levels of social connectivity varied (below average, average, above average, and well above average numbers of friends). Respondents had to indicate which restaurant they thought people in their city would hear about first (i.e., would have the fastest diffusion). Eighty-three percent of respondents believed that news of the restaurant talked about by the person with "well above average" connectivity would spread the fastest.

Finally, we asked respondents to indicate on three five-point scales $(1=$ strongly disagree, $5=$ strongly agree) the extent to which they believed that faster diffusion would result from information being shared by a higher-connectivity person ("People with many social connections/friends are important for spreading information," "When a person with many social 
connections shares news with others it spreads quickly," and "If a person with many social connections shares information it will spread like wildfire"). The means (SDs) for the three items were $4.05(.72), 4.11(.60)$, and $3.98(.75)$, and the percentages indicating "agree" or "strongly agree" on these scales were $83 \%, 92 \%$, and $79 \%$. Thus, based on this pretest, people appear a have a lay belief about fast diffusion and connectivity consistent with our conceptualization.

Our proposed mechanism for encouraging or "nudging" transmissions to higherconnectivity receivers is based on taking advantage of this lay belief. Specifically, it involves linking a regular consumer's expected transmission utility to the number of other people who will possess the transmitted information (i.e., the eventual number of "informed" people). In this situation, transmitters should have an incentive to favor higher-connectivity friends as receivers. This is related to prior research on interdependent consumer choice in which one's utility is influenced by, or is partly a function of, others' utilities (e.g., Yang and Allenby 2003). More generally, it is based on the (positive) network externality concept in economics, which is where a "network good" (i.e., with a positive externality) is more valuable to a consumer as the number of consumers owning or using that good increases (e.g., Economides 1996; Katz and Shapiro 1985; Leibenstein 1950). ${ }^{4}$ We consider positive externalities in information that consumers transmit to friends via WOM. If the transmitted information has a positive externality, based on the above logic, transmitters should have a preference for higher-connectivity receivers.

We consider two ways that information can have a positive externality. First, it can be inherent to the good to which the information refers. In other words, the product or service can be a network good, such as a communication technology (e.g., historically, fax machines; recently, social media platforms). When the item is a network good, marketers can encourage

\footnotetext{
${ }^{4}$ Although not considered here, negative externalities are also possible. This occurs if information value decreases as it becomes widely known, creating a transmission disincentive (e.g., Frenzen and Nakamoto 1993).
} 
higher-connectivity receiver selection by making the externality salient. Second, for non-network goods, marketers can use tactics such as promotions to introduce a positive externality. ${ }^{5}$ Instead of rewarding a customer for making WOM transmissions or referrals directly, a promotion-based mechanism could make rewards contingent on macro-level diffusion outcomes. This approach has been used in practice by "daily deal" websites, "flash sales" retailers, and crowdfunding platforms such as Kickstarter. For example, a transmitter might be tempted with a deep discount that is only realized if a minimum number of consumers sign up for that deal. In sum, this gives rise to the following hypothesis:

$\mathrm{H}_{1}$ : Preference for higher-connectivity receivers will be higher when the information to be transmitted has a positive externality.

Also, as discussed earlier, preference for higher-connectivity receivers is expected to be higher under a positive externality because this makes faster macro-level diffusion more important and relevant to transmitters. Thus, the positive effect of a mechanism that makes salient an inherent externality or introduces it through marketing tactics will be mediated by a transmitter's belief in the importance of faster macro-level diffusion. Stated formally:

$\mathrm{H}_{2}$ : The positive effect of a positive-externality mechanism on preference for higherconnectivity receivers will be mediated by transmitter-perceived importance of faster diffusion.

The second condition for a mechanism to make the transmitter's weight on receiver connectivity more positive is that there must be a sense of urgency or limited time for achieving fast diffusion. Urgency or limited time is posited to be a moderator of the effects predicted in $\mathrm{H}_{1}$ and $\mathrm{H}_{2}$. If it does not matter how long it will take for information to spread, diffusion speed will

\footnotetext{
${ }^{5}$ Although it was a negative externality, the scarce price discount in Frenzen and Nakamoto (1993) is an example of how externalities can be introduced through marketing tactics.
} 
not be important to transmitters. Accordingly, when faster diffusion is unimportant (even if diffusion in general is important), a positive-externality mechanism should not strongly influence a transmitter's preference for higher-connectivity receivers. Specifically, if the outcome is not urgent then we would not expect faster diffusion to be affected by a positive-externality mechanism, which in turn would mean that the mechanism does not impact preference for higher-connectivity receivers. Stated formally:

$\mathrm{H}_{3}$ : The positive effect of a positive-externality mechanism on preference for higherconnectivity receivers, mediated by transmitter-perceived importance of faster diffusion, will be enhanced when transmitters feel a sense of urgency for the diffusion outcome.

\subsection{Summary and Overview of Studies}

Our conceptual framework is summarized in Figure 1. We propose that an important firm-desired macro-level goal for consumer WOM marketing campaigns-faster information diffusion or social mobilization — can be made more likely through the use of marketer-initiated mechanisms that encourage or "nudge" WOM-transmitting customers to select higherconnectivity friends as receivers. The proposed mechanisms make transmissions to higherconnectivity friends more likely by influencing how important faster diffusion is to transmitters, particularly when transmitters feel a sense of urgency.

\section{[INSERT FIGURE 1 ABOUT HERE]}

We test our hypotheses with three experiments and a simulation. Study 1 tests $\mathrm{H}_{1}$ in the context of a "refer a friend" WOM campaign setting where the externality is inherent and is made salient. Study 2 replicates this in a different context where the externality is introduced 
through a promotional offer, and tests $\mathrm{H}_{2}$ and $\mathrm{H}_{3}$. Study 3 is a field experiment that replicates a key part of Study 2 in different setting, and provides evidence of a positive relationship between the selection of higher-connectivity receivers and faster diffusion. Finally, Study 4 is a simulation that demonstrates how making it more likely that higher-connectivity receivers are chosen can lead to faster macro-level diffusion, and also compares this approach to a common approach used in practice, hub seeding.

\section{Study 1}

This study tests $\mathrm{H}_{1}$ by showing that a positive-externality mechanism can increase the social connectivity of nominated receivers in a "refer a friend" WOM campaign. The mechanism makes an inherent externality salient, and compares this to a control condition where the externality is also present but not made salient. This allows us to test the effectiveness of making an inherent externality salient in the case of network goods (non-network goods are considered in Study 2). This is a field experiment in which participants made actual transmission decisions.

\subsection{Design and Procedure}

Sixty undergraduate students at the University of Pittsburgh participated in this field experiment for course credit as part of an hour-long laboratory session. At the end of the session (after completing other tasks that were unrelated to this experiment), participants were asked to provide their email address for a mailing list that would be used to advertise future sessions in a new lab facility that allowed them to earn cash instead of course credit. To increase the realism of the task, before exposure to the manipulation, participants were also asked (i) how interested they were in participating in more lab sessions ( $1=$ not at all, $5=$ extremely), (ii) how long these 
sessions should ideally be, (iii) their ideal hourly pay rate, and (iv) how often they want to participate $(1=$ never, $7=$ daily $)$. Participants were then asked to nominate (i.e., refer) a friend who was also a student at the university who they thought would also like to be on the email list (i.e., who they thought would be relevant). We then asked some questions about this person, including how socially well connected this friend is $(1=$ not at all, $5=$ extremely socially connected), which was used as the dependent variable. Other questions were asked to reduce potential for hypothesis guessing: (i) college year, (ii) business major (yes/no), (iii) how well this person is known to the participant $(1=$ not at all, $5=$ extremely well), and (iv) will they be interested in paid lab sessions (yes/no).

Participants were given a cover story, which varied according to the condition to which participants were randomly assigned. The experiment used a 2(control, externality) betweensubjects single-factor design. In the control condition, participants were told to nominate a friend because "We need your help spreading the word [about the new paid-participation opportunity]." In the externality condition, participants were told the same, plus reminded that unless enough students signed up for these paid-participation experiment sessions, it was unlikely that they would have the opportunity to earn extra money because sessions would not be scheduled or, if they were, could be canceled because the minimum number of required participants might not be achieved (see Web Appendix B). Our intention with this manipulation was to make salient an inherent positive externality that existed in both conditions, i.e., the opportunity to make extra money in this lab always depends on sessions running, which depends on having enough students in the participant pool. This is a subtle externality and without being explicitly made aware of it, people would be unlikely to think of it themselves. Thus, this study is a fairly conservative test of $\mathrm{H}_{1}$. 


\subsection{Results}

Consistent with $\mathrm{H}_{1}$, an ANOVA revealed that connectivity of the nominated friend is

higher in the externality condition $\left(\mathrm{M}_{\text {externality }}=3.84 \mathrm{vs.} \mathrm{M}_{\text {control }}=3.21, F(1,58)=7.01, p=.01\right)$. Thus, a mechanism that simply draws attention to an inherent externality can be effective. Explicit incentive mechanisms are therefore not always necessary, although they can be effective when marketing non-network goods, as we show in Study 2.

In addition to receiver connectivity, we measured two other receiver characteristics to mask the dependent variable and to minimize potential demand bias. Our manipulation had no effect on whether chosen receivers were expected to be interested in participating in lab studies $\left(\chi^{2}(1)<1, p=.55\right.$; overall, $88 \%$ of participants said their receiver would be interested $)$. The other measure was how well the participant felt they knew their chosen receiver (i.e., tie strength). This was affected by the manipulation, though not as strongly as the dependent variable was $\left(\mathrm{M}_{\text {externality }}=4.44\right.$ vs. $\mathrm{M}_{\text {control }}=3.96 ;(F(1,58)=4.14, p=.046)$. Importantly, when this variable was included as a covariate in the main analysis, the positive effect of the manipulation on receiver connectivity remained significant $(F(1,57)=4.42, p=.039)$.

A possible limitation of this study is how receiver connectivity was measured, i.e., as the transmitter's perception of their nominated friend's social connectivity. Prior research suggests that perceptions sometimes do not capture reality (e.g., Krackhardt 1987). Social connectivity measurement research uses two types of measures: perceptual (our approach) and sociometric (using network data) (e.g., Parkhurst and Hopmeyer 1998). Sociometric measures are thought to be superior because they are based on the actual number of friends people have, but accessing such data is often impossible. Thus, for practical reasons, researchers often rely on perceptions. In any case, if a transmitter considers connectivity when selecting a receiver, it will be 
perceptional, since regular people do not have accurate sociometric data on their friends. Further, popularity research suggests that perceptions of others' social connectivity are decent indicators of sociometric connectivity (i.e., these measures are moderately-to-strongly positively correlated; e.g., Kleck, Reese, Ziegerer-Behnken, and Sundar 2007; Parkhurst and Hopmeyer 1998).

\section{Study 2}

The previous study showed how making an inherent externality salient causes transmitters to be more likely to select higher-connectivity receivers, consistent with $\mathrm{H}_{1}$. The purpose of this next study is to show a similar effect for a non-network good where the externality is introduced (vs. made salient) through a marketing promotion. Additionally, this study tests $\mathrm{H}_{2}$ and $\mathrm{H}_{3}$; i.e., it provides evidence that higher-connectivity receivers are preferred when a positive-externality mechanism is used because this increases transmitters' perceptions of the importance of fast diffusion, particularly when this is urgent.

\subsection{Design and Procedure}

Three-hundred and sixty-nine U.S. members of Amazon Mechanical Turk (mean age = 33.6 years, $42.6 \%$ female) participated in this experiment for a nominal monetary payment. Participants considered a scenario where they received an email from Amazon announcing a "flash sale" on Amazon gift cards, where cards with face values up to $\$ 250$ could be purchased at $50 \%$ off. Participants were randomly assigned to conditions in a 2(control, externality) x 2(not urgent, urgent) between-subjects design.

Since the product here is a non-network good, the manipulation introduced an externality instead of making an inherent externality salient. To be realistic and familiar to participants, the 
manipulation was designed to be similar to methods used to encourage WOM by crowdfunding platforms such as Kickstarter (i.e., where a minimum amount of funding is needed to be successful) and “daily deals" services such as Groupon. In the control condition, participants were told that there was "no catch" to this offer and they could take advantage of it by using a discount code at any time during the offer period (see Web Appendix B). The offer period was seven days in the not-urgent condition, and one day in the urgent condition. In the externality condition, participants were told that there was a "catch" such that for the discount to be received a "minimum of 50,000 people must make a gift card purchase" within the offer period (and, to be realistic, it was stated in the externality condition that if this minimum was not met there was no charge). After the email, participants indicated how likely they would be, on a 0-100 percent scale, to share this offer with a higher-connectivity friend. This was the dependent variable.

Next, we measured the mediator, desire for fast diffusion, with two seven-point Likertscaled items ( 1 = strongly disagree, 7 = strongly agree; "I want many other people to know about this offer as quickly as possible" and "I want many other people to share this offer soon"; $r=$ .87). Included with these two items (in random presentation order) we measured desire for widespread (but not necessarily faster) diffusion using three items ("It is important to me that many other people redeem this offer," "I want many other people to know about this offer" and "I do not care if many other people redeem this offer" [reversed]; $\alpha=.85$ ), and desire for faster (but not necessarily widespread) diffusion with a single item ("I want word of this offer to spread as quickly as possible"). These additional items were included to enable us to show that the externality effect on the probability of transmitting to a higher-connectivity friend is mediated by desire for information to spread widely and quickly, not just widely or quickly. The three 
composite items for the mediator and the two alternatives were, as expected, highly positively correlated $(r \mathrm{~s}>.86)$, despite the important but subtle conceptual distinctions between them.

\subsection{Results}

First, we compared mean higher-connectivity receiver transmission probabilities between the control and externality conditions. Consistent with $\mathrm{H}_{1}$, an ANOVA revealed a positive effect of externality $\left(\mathrm{M}_{\text {externality }}=73.57\right.$ vs. $\left.\mathrm{M}_{\text {control }}=62.22, F(1,367)=10.48, p=.001\right)$. When urgency was included in the model and interacted with externality, the main effect of externality was again positive and significant $(F(1,385)=10.55, p=.001)$. The urgency main effect, however, was not significant $(p=.35)$. The externality-by-urgency interaction was also not significant, but somewhat stronger $(F(1,385)=2.33, p=.13)$. Contrasts testing for the simple effect of externality at each level of urgency showed a significant positive simple effect of externality when the faster-diffusion outcome was urgent ( 1 day; $\mathrm{M}_{\text {externality }}=77.94$ vs. $\mathrm{M}_{\text {control }}=61.23, F(1$, $365)=11.47, p=.001)$. When the outcome was not urgent, the simple effect of externality was not significant $\left(7\right.$ days; $\mathrm{M}_{\text {externality }}=69.30$ vs. $\left.\mathrm{M}_{\text {control }}=63.26, F(1,365)=1.47, p=.23\right)$.

We next tested the moderated-mediation process $\left(\mathrm{H}_{2}\right.$ and $\left.\mathrm{H}_{3}\right)$. We expected that the positive externality effect on preference for transmitting to higher-connectivity receivers occurs through an increased desire for faster diffusion, and that this effect would be stronger when the outcome is more urgent. A conditional indirect effects test (Hayes 2013, model 8) supported our predictions. There was a significant positive indirect effect of the externality on the probability of transmitting to a higher-connectivity friend through desire for fast diffusion. This was stronger in the urgent condition (indirect effect of externality $=12.85, \mathrm{SE}=1.93,95 \%$ C.I. $=[9.16,16.73]$ vs. not-urgent indirect effect of externality $=6.80, \mathrm{SE}=1.79,95 \%$ C.I. $=[3.31,10.33])$. 
Finally, we attempted to distinguish between a desire for widespread (but not necessarily faster) diffusion and a desire for faster (but not necessarily widespread) diffusion as mediators, using the two additional constructs measured alongside the desire for faster diffusion mediator. We repeated the moderated-mediation analysis but replaced the mediator with these two alternatives operating in parallel. Given that these alternative mediators are highly correlated $(r=$ .86), and they are both highly correlated with the main mediator, we expected to find that both operated as mediators in a manner consistent with the above-reported results. This was the case (widespread but not fast indirect effect of interaction $=.92, \mathrm{SE}=.49,95 \%$ C.I. $=[.16,2.13]$; fast but not widespread indirect effect of interaction $=1.76, \mathrm{SE}=.95,95 \%$ C.I. $=[.04,3.80])$. Consistent with our theory, the moderated-mediation was stronger through faster (but not necessarily widespread) diffusion than through widespread (but not necessarily faster) diffusion.

\section{Study 3}

The previous study showed how an externality can be introduced through a marketing tactic, which can make consumers more likely to transmit to higher-connectivity friends, consistent with $\mathrm{H}_{1}$. Additionally, we showed how this occurs through a desire for fast diffusion, particularly when the outcome is reasonably urgent, in line with $\mathrm{H}_{2}$ and $\mathrm{H}_{3}$. The purpose of this next study is to (i) provide further process evidence in support of $\mathrm{H}_{2}$, (ii) do this using a different mediator operationalization and in a different context, and (iii) show a link between the increased likelihood of transmitters selecting higher-connectivity receivers and macro-level faster diffusion. This study is a field experiment. 


\subsection{Design and Procedure}

Ninety-seven U.S. members of Amazon Mechanical Turk (mean age $=32.6$ years, $40.2 \%$ female) participated in this study for a nominal monetary payment. The context was a public health communication in the form of a public service announcement (PSA) from the U.S. Centers for Disease Control about the importance of getting a seasonal influenza vaccination (i.e., flu shot). Participants were assigned to one of two conditions in a 2(control, externality) between-subjects single-factor design. Faster diffusion was urgent in both conditions (i.e., we did not manipulate urgency in this study). The study was conducted in late September (before the typical U.S. flu season has begun). To create a sense of urgency, participants were told that the U.S. Centers for Disease Control expected the flu season to start early, and were therefore advised to get their flu shot by early October (i.e., within one or two weeks of the study). Importantly, participants were told that they could help reduce the risk of a flu outbreak by telling a friend about the importance of getting a flu shot, and were asked to inform a friend by emailing them a link to the official government flu information website, Flu.gov. Each condition had its own unique link that participants were asked to share with a friend that could be tracked (for measuring macro-level diffusion speed), and participants were aware that this link was unique and trackable.

The mechanism for manipulating externality was somewhat similar to the manipulation used in Study 2. In the externality condition, participants were offered an incentive for emailing this link to a friend that was designed to encourage them to email a higher-connectivity friend. The incentive was a bonus payment (double their payment for participating in the study) if 50 or more people visited Flu.gov via their unique link over the next seven days. There was no such incentive offered in the control condition. After reading the PSA, participants were given a few 
minutes to think about one friend to whom they wanted to email the trackable link. A webpagebased form was used for sending the email. Participants entered their friend's email address and a message. The software automatically sent this message via email to the designated receiver's address, included the link to Flu.gov in the email, and tracked email sending and clicks on links.

After sending the email, participants responded to items similar to those used in Study 1. Receiver connectivity, the dependent variable, was measured similar to Study 1, with two fivepoint items ("How social is this person?" $1=$ not at all, has very few friends/connections, $5=$ extremely, has a very large number of friends/connections; "How socially well connected is this person relative to other people you know?" $1=$ well below average social connectivity, $5=$ well above average social connectivity; $r=.76)$. We also measured two additional receiver characteristics: tie strength (five-point scale, "How well do you know this person?" $1=$ not at all, $5=$ extremely well), and perceived receiver interest (five-point scale, "Do you think this person will be interested in hearing about flu vaccinations?" $1=$ definitely no, $5=$ definitely yes).

Perceived receiver interest was used as a mediating variable in this study, as an indirect measure of the underlying conceptual mediating construct, desire for faster diffusion. Although this is quite different from the measure used in Study 2, we employed this indirect approach for an important reason. The measure in Study 2 refers directly to the macro-level faster-diffusion construct, which, although it matches our conceptualization, might have been difficult for participants to conceive of given that they think at a micro, not macro, level in this setting. Thus, in this study we tried to measure the underlying conceptual mediator in a manner that was easier and more relatable for participants. The specific measure-perceived receiver interest-is a characteristic that transmitters plausibly think of when selecting receivers. Importantly, this is likely to be a strong correlate of the underlying macro-focused mediator, desire for faster 
diffusion. This is because if the faster-diffusion outcome is important to a transmitter, it should be important to them that the receiver is interested in the topic since interest means they are likely to listen, which is necessary if that receiver is to retransmit the information to others (which is needed for faster diffusion).

\subsection{Results}

To test that the positive externality effect on receiver connectivity operates through desire for faster diffusion $\left(\mathrm{H}_{2}\right)$, we performed a conditional indirect effects test (Hayes 2013, model 4). Consistent with $\mathrm{H}_{2}$, the positive externality mechanism had a positive and significant indirect effect on nominated receiver connectivity (indirect effect $=.06, \mathrm{SE}=.04,95 \%$ C.I. $=[.01, .16]$ ).

We did the same analysis with tie strength as the dependent variable. The purpose of this was to show that the externality affects a particular aspect of receiver selection related to the faster-diffusion outcome and not other aspects, such as whether more- or less-close friends are receivers. Based on our conceptualization, tie strength should not be affected because how close a friend a potential receiver is to a transmitter should not matter with respect to a transmitter's perception of their ability to spread information quickly throughout their networks. Consistent with this, we found no evidence suggesting that positive interdependence affects transmitterreceiver tie strength (direct effect $p=.31$; indirect effect $=.02, \mathrm{SE}=.03,95 \%$ C.I. $=[-.02, .09]$ ).

Finally, because participants emailed trackable links we were able to see if macro-level fast diffusion was higher in the positive interdependence condition. Because the incentive said that bonus payments would be made if enough diffusion occurred within a fixed timeframe of seven days, an appropriate measure of macro-level fast diffusion is simply Flu.gov link clicks over seven days (i.e., the link-click or click-through rate; i.e., clicks as a percentage of sent 
links). In the control condition, the link-click rate was $7.02 \%$, which was significantly lower than in the positive interdependence condition, where the rate was $41.94 \%(Z=4.40, p<.001)$. While this result should be treated as tentative because we had no observation of the link-diffusion process beyond the nominated receiver, the difference is convincingly large.

\section{Study 4}

The three previous studies focused on demonstrating how making an externality salient (network goods) or introducing an externality through marketing tactics (non-network goods) can make regular-consumer transmitters more likely to transmit WOM to higher-connectivity receivers. In this study, we show how an increased preference for higher-connectivity receivers can lead to macro-level faster diffusion. This study uses a simulation for this purpose. Our approach is in the tradition of work in the diffusion literature that has used simulations and agent-based modeling (e.g., Goldenberg et al. 2001, 2004; Lusch and Tay 2004; Libai et al. 2013; Rand and Rust 2011).

\subsection{Model of Individual-Level Behavior and WOM Transmission}

Our model follows existing individual-level diffusion models (e.g., Goldenberg et al. 2001, 2004; Toubia, Goldenberg, and Garcia 2014) that are related to the classic Bass (1969) diffusion model. To allow us to test the extent to which a preference for higher-connectivity receivers affects diffusion speed, we included a parameter that represents the weight (i.e., importance) that transmitters put on receiver connectivity when selecting receivers. We used this as a way to model the effect of the externality manipulations used in the previous studies, since making salient or introducing a positive externality had the effect of making receiver connectivity more important to transmitters. 
Consider a population of $N$ people, each of whom is a node in a social network. Consistent with what is commonly found in real-world social networks, we assume that this network is sparse and scale free, meaning that the number of connections nodes have is powerlaw distributed (Barabasi and Albert 1999). Person $i$ (for $i=1, \ldots, N$ ) is connected to $d_{i}>0$ others. Here we operationalize information diffusion as awareness of a product spreading across the network in discrete time $(t=1, \ldots, T)$. Person $i$ 's awareness of the product at the end of period $t$ is state variable $0 \leq a_{i t} \leq 1$, with $a_{i t}=1\left(a_{i t}=0\right)$ meaning that person $i$ is "completely aware" ("completely unaware") of the product. At $t=0, a_{i, 0}=0$ except for a small group of "seeds" who have $a_{\text {seeds }, 0}=1$ to start the diffusion process.

The WOM transmission process involves two steps. First, people with $a_{i, t-1}>0 \mathrm{might}$ transmit WOM in period $t$. Awareness corresponds to the extent to which the product is "top of mind," and the more top of mind a product is, the more likely a person is to transmit WOM about that product at that time. They can, however, choose to "stay silent" despite having nonzero awareness. Transmission is impossible if $a_{i, t-1}=0$ since the person is unaware. Thus, first we decide whether or not person $i$ in period $t$ will transmit, with the rule as follows: if $\operatorname{Bernoulli}\left(a_{i, t-}\right.$ $\left.{ }_{1}\right)=1$ person $i$ transmits in period $t$ (otherwise they will "stay silent"). Then, if Bernoulli $\left(a_{i, t-1}\right)=$ 1, we allow person $i$ in period $t$ to transmit to any of their $d_{i}$ friends. For persons $i$ and $j$ who are connected, the probability that $i$ transmits to $j$ in period $t$ is defined as ptransmit $t_{i j t}=\exp \left(\alpha d_{j}\right) /[1+$ $\exp \left(\alpha d_{j}\right)$ ]. The critical parameter is $\alpha(\geq 0)$, the importance weight that person $i$ puts on friend $j$ 's social connectivity $\left(d_{j}\right)$ when "deciding" if they will transmit the information to them. When $\alpha=$ 0 receiver connectivity does not matter to transmitters; i.e., ptransmit $_{i j t}=.50$. As $\alpha$ increases, ptransmit $_{i j t}$ increases. The transmission rule is as follows: if Bernoulli $\left(\right.$ ptransmit $\left._{i j t}\right)=1$ person $i$ will transmit to person $j$ in period $t$. This is similar to the model in Toubia et al. (2014). 
We then consider how WOM transmission affects selected receivers. To be conservative and realistic, we accommodate the possibility that people do not listen. Receiver $j$ has $d_{j} \geq x_{j t}$ friends, where $x_{j t}$ is the number of $j$ 's friends who chose to transmit to $j$ in period $t$. Following Toubia et al. (2014), the hazard of $j$ becoming completely aware of the product in period $t\left(a_{j t}=\right.$ 1) is preceive $_{j t}=P\left(a_{j t}=1\right)=1 \quad(1 \quad p)(1 \quad q)^{x_{j t}}$, where the parameters $p$ and $q$ are coefficients of “external" influence (e.g., advertising) and "internal" influence (WOM) similar to those in the Bass diffusion model. If Bernoulli $\left(\right.$ preceive $\left._{t}\right)=1$, receiver $j$ is aware of the product at the end of period $t$ (i.e., $a_{j t}=1$ ). Finally, for realism, we allow awareness to decrease between periods, which captures forgetting, memory decay, distractions, or the presence of other things to talk about. Thus, we multiply end-of-period awareness $a_{i t}$ by $(1-\delta)$, where $0 \leq \delta<1$, to arrive at an end-of-period awareness level for each person that carries over to the start of the next period.

\subsection{Simulation Design}

This model of WOM transmission behavior was used to simulate diffusion processes over a $N=1,000$ social network with a realistic structure. The network was randomly generated, was "scale free" with exponent parameter $=2.19$ (which is in the typical 2-3 range; Barabasi and Albert 1999), and had mean degree of 3.99 (i.e., on average, a person in this network has approximately four friends). Thus, the network used was typical of real-world social networks, particularly offline social networks. The simulation was a full-factorial experiment in which five parameters were varied. First, and most important, weight on receiver connectivity, $\alpha=\{0, .20$, $.40, .60, .80,1.00,1.20\}$. Second, coefficient of external influence, $p=\{0, .01, .02, .03, .04\}$. Third, coefficient of internal influence, $q=\{.10, .20, .30, .40\}$. The $p$ and $q$ ranges follow Sultan, Farley, and Lehmann (1990). Fourth, awareness decay, $\delta=\{0, .20\}$. Fifth, number of seed nodes 
completely aware at $t=0, N_{\text {seeds }}=\{5,10\}$, which corresponds to seeding $.5 \%$ and $1 \%$ of the population as is typical in seeding campaigns. Seeds were selected randomly in each run. The full factorial design had 560 cells and we ran the simulation five times per cell for robustness (i.e., for a total of 2,800 simulation runs). Each run covered $T=365$ periods, mimicking daily diffusion over one year.

A second set of simulations was run with "hub seeding" instead of random seed seeding (e.g., Hinz et al. 2011). This allowed us to compare results to a situation in which a firm can directly target higher-connectivity individuals as seeds. Hubs were identified using Goldenberg et al.'s (2009) criterion of a node having degree greater than or equal to three standard deviations above the network's mean degree. We randomly sampled $N_{\text {seeds }}$ from the set of identified hub nodes. The mean degree of the seeds was 3.99 when randomly selected from all nodes versus 40.16 when randomly selected from the subset of hub nodes. Since typical hub seeding does not employ the externality-based mechanism we focus on, $\alpha=0$ in the hub seeding condition.

\subsection{Results}

The dependent variable in our analysis is diffusion speed, measured for each simulation run as the maximum number of people (nodes) who became aware of the product during the simulation divided by the number of periods it took to reach this maximum. Diffusion speed was regressed on each of the five varied model parameters. We included a random effect to control for multiple simulation runs for each combination of model parameters. Overall, we used 2,800 observations from 560 cells.

As expected, the weight on receiver connectivity $(\alpha)$ had a significant positive effect on diffusion speed $(b=9.44, t=19.28, p<.001)$. All other varied parameters had a significant 
effect on diffusion speed, except for seed size. Mean diffusion speeds at each level of this parameter are plotted in Figure 2 (averaging over the levels of the other parameters; error bars are one standard error above/below the means). As this figure shows, diffusion speed increases as transmitters' preference for receiver connectivity gets stronger. This demonstrates that the externality-based mechanisms tested in the previous studies that affect transmitters' receiver selection behaviors can lead to faster information diffusion at the macro level. As a robustness check, we also ran this analysis for the numerator and denominator of diffusion speed separately. The results were consistent: increasing weight on receiver connectivity increases the number of people who become aware in a set period of time, and decreases time taken to reach maximum awareness or time to peak.

\section{[INSERT FIGURE 2 ABOUT HERE]}

We compared this to hub seeding. The dotted horizontal line on Figure 2 represents the case of hub seeding with zero weight on receiver connectivity $(\alpha=0)$, which is good representation of standard practice in seeded WOM campaigns. Hub seeding outperforms nonhub (i.e., random, regular consumer) seeding only if seeds put zero weight on receiver connectivity $(t=2.00, p=.05)$. However, when randomly selected, regular-consumer seeds put weight on receiver connectivity, hub seeding is inferior in terms of diffusion speed, even for small values of $\alpha$. This is important because it means that strong externality mechanisms are not required. More broadly, it shows that externality-based inducements can be superior to hub seeding as a way to speed-up diffusion. 


\section{Discussion and Implications}

Given the importance of customer-driven WOM communications to marketers, it is not surprising that multiple streams of research consider various facets of WOM marketing and, most relevant to the current research, WOM-based referrals and seeded WOM campaigns. Despite the large extant body of research, few studies have considered consumers' transmission behaviors with respect to types of receivers chosen. Moreover, using mechanisms or incentives to encourage regular WOM-transmitting consumers to select certain types of friends as receivers has not been previously explored. Our results show how marketers can move beyond merely encouraging customers to transmit WOM to anyone by implementing straightforward mechanisms to get consumers to target their transmissions at a particular type of receiver. In other words, it is possible to "nudge" consumers to select a certain type of receiver-higherconnectivity friends, in this case — by leveraging network effects with simple marketing tactics or incentive-based promotions. We also showed that this occurs because transmitters then have a stronger desire for fast diffusion, which suggests that positive-externality mechanisms can be used to align certain aspects of individuals' transmission objectives with a firm's goals (in this case, faster diffusion). Theoretically, this implies that effective mechanisms for influencing consumers' WOM transmission behaviors work by getting transmitters to think about — and buy into - the firm's intentions and objectives. This does not mean that complete "goal alignment" is necessary, but rather that a mechanism will operate well if it gets customers to be cognizant of (and in favor of) the firm-desired consequence of their WOM-transmission actions.

We focused specifically on encouraging micro-level receiver selection decisions that can help give rise to faster information diffusion (or "high-speed diffusion"). This is an important marketing objective when relying on WOM to spread information about brands, products, and 
services. A key implication of the current research is that marketers do not need to go to great lengths to identify, recruit, and active "special" consumers - such as so-called "hubs" with high levels of social connectivity. Marketers can instead seed information with regular consumers (or randomly) and use externality-based mechanisms such as those tested here to encourage transmitter behaviors that will make faster diffusion more likely. While suggested in the literature on diffusion over networks (e.g., Adamic et al. 2001; Goldenberg et al. 2009) and in the viral seeding literature (e.g., Hinz et al. 2011), it helps to have higher-connectivity people involved, marketers can use regular consumers to get information to these individuals instead of seeking them directly. Having higher-connectivity people as receivers is important when marketers cannot easily directly reach these people and encourage them to transmit WOM, which is often the case in practice.

Our approach using positive-externality mechanisms that either make an inherent externality salient for network goods, or introduce it through marketing tactics for non-network goods, is novel and can be easily used by marketers. The manipulations we used could be used in practice with, at most, minor modifications. Our findings also present marketers with a viable alternative to directly targeting higher-connectivity customers through viral "hub" seeding practices, which in many cases is either impossible or expensive and might not work because hubs could be less susceptible to influence, or sought-after by many firms at the same time. The approach has the advantage of being usable with regular customers (i.e., anyone), involves simple "tweaks" to existing marketing messaging or promotional tactics, and is efficient because it takes advantage of consumers' knowledge of their own social networks which is likely superior to the knowledge possessed by marketers of consumers' social connections. Additionally, although not tested in our studies, we expect our approach to be complementary to 
other efforts to generate faster diffusion, such as hub seeding or using seeds with other special characteristics, such as "opinion leaders" or "influencers."

Theoretically, an important aspect of the current research is shifting the focus on transmitter selection ("Who is the transmitter?") in extant literature to receiver selection ("To whom is information transmitted?"), and, critically, how marketers can influence regular consumers' receiver selection decisions in desirable ways. Practically, the externality-based approach employed here is straightforward and easily implementable by marketers. While it is common to offer incentives of various kinds to consumers in exchange for WOM transmissions, the status quo is concerned with getting the transmissions to take place, irrespective of who the receivers are. This is not suboptimal because it will not necessarily expedite information diffusion. Our approach is therefore especially useful when faster diffusion is desired, which is likely to be the case most of the time.

The current work is not without limitations. First, we focused entirely on receiver connectivity and how to increase transmissions to higher-connectivity friends. Although we demonstrated that it is possible to use externality-based mechanisms to achieve this, it would be interesting for future research to explore how other social transmission behaviors can be encouraged through other mechanisms. Second, our approach did not take into account other receiver characteristics that marketers might look for and that might help drive other macro-level diffusion outcomes of interest to marketers. For example, the extent to which a person is an “influential” or "opinion leader" might be important, although some studies suggest otherwise (Iyengar et al. 2011; Van den Bulte and Lilien 2001; Watts and Dodds 2007). Future research could consider interpersonal influence as another relevant receiver characteristic. Third, we did not consider potential moderators to these effects, such as product category or individual 
differences, which could be a useful direction for future research. Fourth, we relied on participants' perceptions of receiver connectivity instead of sociometric connectivity. As discussed earlier (in Study 1), people often rely on perceptions of how connected friends are since they do not have better information. Even though "objective" indicators of social connectivity exist for people in online social networks such as Facebook and Twitter, how many friends or followers one has is not a perfect measure of one's social connectivity since these measures are biased upwards (e.g., due to fake accounts). Finally, although our focus was specifically on receiver connectivity as a "target" characteristic for the mechanism, it would be very interesting for future research to consider a mechanisms for targeting other desirable receiver characteristics.

In conclusion, four studies (two field experiments, a scenario-based experiment, and a simulation) demonstrated the efficacy of using positive externality-based mechanisms for encouraging or "nudging" regular consumers' WOM transmissions such that they were more likely to prefer, and therefore select, higher-connectivity friends as receivers. We considered this across a variety of settings, for network and non-network goods, and linked the micro-level receiver selection behavior to macro-level faster diffusion. Our findings extend the WOM marketing literature by introducing an approach for encouraging transmissions to specific types of consumers to accelerate diffusion that is a viable alternative to directly targeting higherconnectivity consumers as seeds in WOM campaigns. Hopefully future research will build on our approach in order to make further progress in this important area. 


\section{References}

Adamic, Lada A., Rajan M. Lukose, Amit R. Puniyani, and Bernardo A. Huberman (2001), "Search in Power-law Networks," Physical Review E, 64, 046135.

Barasch, Alixandra and Jonah Berger (2014), "Broadcasting and Narrowcasting: How Audience Size Affets What People Share,” Journal of Marketing Research, 51 (3), 286-299.

Berger, Jonah (2011), “Arousal Increases Social Transmission of Information,” Psychological Science, 22 (7), 891-893.

(2014), "Word of Mouth and Interpersonal Communication: A Review and Directions for Future Research," Journal of Consumer Psychology, 24 (4), 586-607.

Biyalogorsky, Eyal, Eitan Gerstner, and Barak Libai (2001), "Customer Referral Management: Optimal Reward Programs,” Marketing Science, 20 (1), 82-95.

Chen, Zoey and Jonah Berger (2013), "When, Why, and How Controversy Causes Conversation," Journal of Consumer Research, 40 (3), 580-593.

Chevalier, Judith A. and Dina Mayzlin (2006), "The Effect of Word of Mouth on Sales: Online Book Reviews," Journal of Marketing Research, 43 (3), 345-354.

Dodds, Peter S., Roby Muhamad, and Duncan J. Watts (2003), "An Experimental Study of Search in Global Social Networks,” Science, 301 (5634), 827-829.

Economides, Nicholas (1996), “The Economics of Networks," International Journal of Industrial Organization, 14 (6), 673-699.

Feick, Lawrence F. and Linda L. Price (1987), “The Market Maven: A Diffuser of Marketplace Information,” Journal of Marketing, 51 (1), 83-97.

Frenzen, Jonathan and Kent Nakamoto (1993), "Structure, Cooperation, and the Flow of Market Information," Journal of Consumer Research, 20 (3), 360-375.

Godes, David and Dina Mayzlin (2004), "Using Online Conversations to Study Word-of-Mouth Communication, Marketing Science, 23 (4), 545-560.

- and - (2009), "Firm-Created Word-of-Mouth Communication: Evidence from a Field Test," Marketing Science, 28 (4), 721-739.

Goldenberg, Jacob, Barak Libai, and Eitan Muller (2001), “Talk of the Network: A Complex Systems Look at the Underlying Process of Word-of-Mouth," Marketing Letters, 12 (3), 211-223.

(2004), “Complex yet Simple: Cellular Automata as Enabling Technology in

Marketing Research.” In Assessing Marketing Strategy Performance, Christine Moorman and Donald

R. Lehman (eds.), Cambridge, MA: Marketing Science Institute.

- Sangman Han, Donald R. Lehmann, and Jae Weon Hong (2009), "The Role of Hubs in the Adoption Process," Journal of Marketing, 73 (2), 1-13. 
Haenlein, Michael and Barak Libai (2013), "Targeting Revenue Leaders for a New Product," Journal of Marketing, 77 (3), 65-80.

Hayes, Andrew F. (2013), Introduction to Mediation, Moderation, and Conditional Process Analysis: A Regression-Based Approach, New York, NY: The Guilford Press.

Hinz, Oliver, Bernd Skiera, Christian Barrot, and Jan U. Becker (2011), "Seeding Strategies for Viral Marketing: An Empirical Comparison," Journal of Marketing, 75 (6), 55-71.

$\mathrm{Hu}$, Yangsong and Christophe Van den Bulte (2014), "Nonmonotonic Status Effects in New Product Adoption," Marketing Science, 33 (4), 509-533.

Iyengar, Raghuram, Christophe Van den Bulte, and Thomas W. Valente (2011), "Opinion Leadership and Social Contagion in New Product Diffusion,” Marketing Science, 30 (2), 195-212.

$\ldots, \ldots$, and Jae Young Lee (2014), "Social Contagion in New Product Trial and Repeat," working paper, University of Pennsylvania.

Katz, Michael L. and Carl Shapiro (1985), "Network Externalities, Competition, and Compatibility," American Economic Review, 75 (3), 424-440.

Kleck, Christine, Christen Reese, Dawn Ziegerer-Behnken, and S. Shyam Sundar (2007), "The Company You Keep and the Image You Project: Putting Your Best Face Forward in Online Social Networks," Proceedings of the Annual Meeting of the International Communication Association.

Krackhardt, David (1987), “Cognitive Social Structures,” Social Networks, 9, 109-134.

Kumar, V., J. Andrew Petersen, and Robert P. Leone (2007), “How Valuable Is Word of Mouth?” Harvard Business Review, October.

, Lerzan Aksoy, Bas Donkers, Rajkumar Venkatesan, Thorsten Wiesel, and Sebastian Tillmanns (2010), "Undervalued or Overvalued Customers: Capturing Total Customer Engagement Value," Journal of Service Research, 13 (3), 297-310.

Lamberton, Cait and Andrew T. Stephen (2016), "A Thematic Exploration of Digital, Social Media, and Mobile Marketing Research's Evolution from 2000 to 2015 and an Agenda for Future Research,” working paper, University of Oxford Saïd Business School.

Leibenstein, H. (1950), "Bandwagon, Snob, and Veblen Effects in the Theory of Consumers' Demand," Quarterly Journal of Economics, 64 (2), 183-207.

Lerman, Kristina, Prachi Jain, Rumi Ghosh, Jeon-Hyung Kang, and Ponnurangam Kumaraguru (2013), "Limited Attention and Centrality in Social Networks," working paper, arXiv: 1303.4451v1.

Libai, Barak, Ruth Bolton, Marnix Bügel, Ko de Ruyter, Oliver Götz, Hans Risselada, and Andrew T. Stephen (2010), “Consumer-to-Consumer Interactions," Journal of Service Research, 13 (3), 267-282.

Mason, Winter and Duncan J. Watts (2009), "Financial Incentives and the 'Performance of Crowds'," ACM Proceedings of KDD-HCOMP. 
Parkhurst, Jennifer T. and Andrea Hopmeyer (1998), "Sociometric Popularity and Peer-Perceived Popularity: Two Distinct Dimensions of Peer Status," Journal of Early Adolescence, 18 (2), 125-144.

Pickard, Galen, Wei Pan, Iyad Rahwan, Manuel Cebrian, Riley Crane, Anmol Madan, and Alex Pentland (2011), “Time-Critical Social Mobilization,” Science, 334 (6055), 509-512.

Rutherford, Alex, Manuel Cebrian, Sohan Dsouza, Esteban Moro, Alex Pentland, and Iyad Rahwan (2013a), "Limits of Social Mobilization," Proceedings of the National Academy of Sciences of the United States of America, 110 (16), 6281-6286.

_- _ Iyad Rahwan, Sohan Dsouza, James McInerney, Victor Naroditskiy, Matteo Venanzi, Nicholas R. Jennings, J. R. deLara, Eero Wahlstedt, and Steven U. Miller (2013b), "Targeted Social Mobilization in a Global Manhunt," PLOS One, 8 (9), e74628.

Ryu, Gangseog and Lawrence Feick (2007), “A Penny for Your Thoughts: Referral Reward Programs and Referral Likelihood,” Journal of Marketing, 71 (1), 84-94.

Schmitt, Philipp, Bernd Skiera, and Christophe Van den Bulte (2011), "Referral Programs and Customer Value," Journal of Marketing, 75 (1), 46-59.

Spiller, Stephen A., Gavan J. Fitzsimons, John G. Lynch, Jr., and Gary H. McClelland (2013), "Spotlights, Floodlights, and the Magic Number Zero: Simple Effects Tests in Moderated Regression," Journal of Marketing Research, 50 (2), 277-288.

Stephen, Andrew T. and Jeff Galak (2012), "The Effects of Traditional and Social Earned Media on Sales: A Study of a Microlending Marketplace,” Journal of Marketing Research, 49 (October), 624-639.

_ (2016), "The Role of Digital and Social Media Marketing in Consumer Behavior," Current Opinion in Psychology, 10 (August), 17-21.

Toubia, Olivier and Andrew T. Stephen (2013), "Intrinsic vs. Image-related Utility in Social Media: Why Do People Contribute Content to Twitter?” Marketing Science, 32 (3), 368-392.

Trusov, Michael, Randolph E. Bucklin, and Koen Pauwels (2009), "Effects of Word-of-Mouth Versus Traditional Marketing: Findings from an Internet Social Networking Site," Journal of Marketing, 73 (September), 90-102.

Van den Bulte, Christophe and Gary L. Lilien (2001), "Medical Innovation Revisited: Social Contagion versus Marketing Effort,” American Journal of Sociology, 106 (5), 1409-1435.

Verlegh, Peeter W. J., Ganseog Ryu, Mirjam A. Tuk, and Lawrence Feick (2013), "Receiver Responses to Rewarded Referrals: The Motive Inferences Framework," Journal of the Academy of Marketing Science, 41, 669-682.

Watts, Duncan J. and Peter S. Dodds (2007), "Influentials, Networks, and Public Opinion Formation," Journal of Consumer Research, 34 (4), 441-458. 
Figure 1

Conceptual Framework

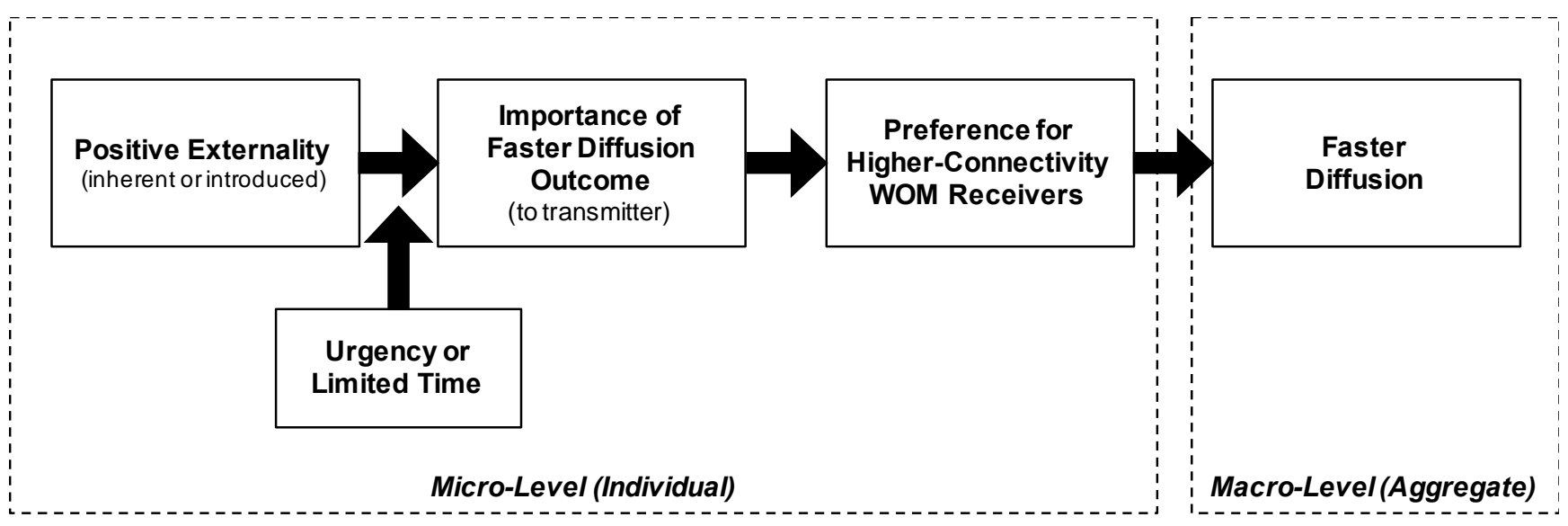


Figure 2

Diffusion Speeds For Different Weights on Receiver Connectivity in Study 4

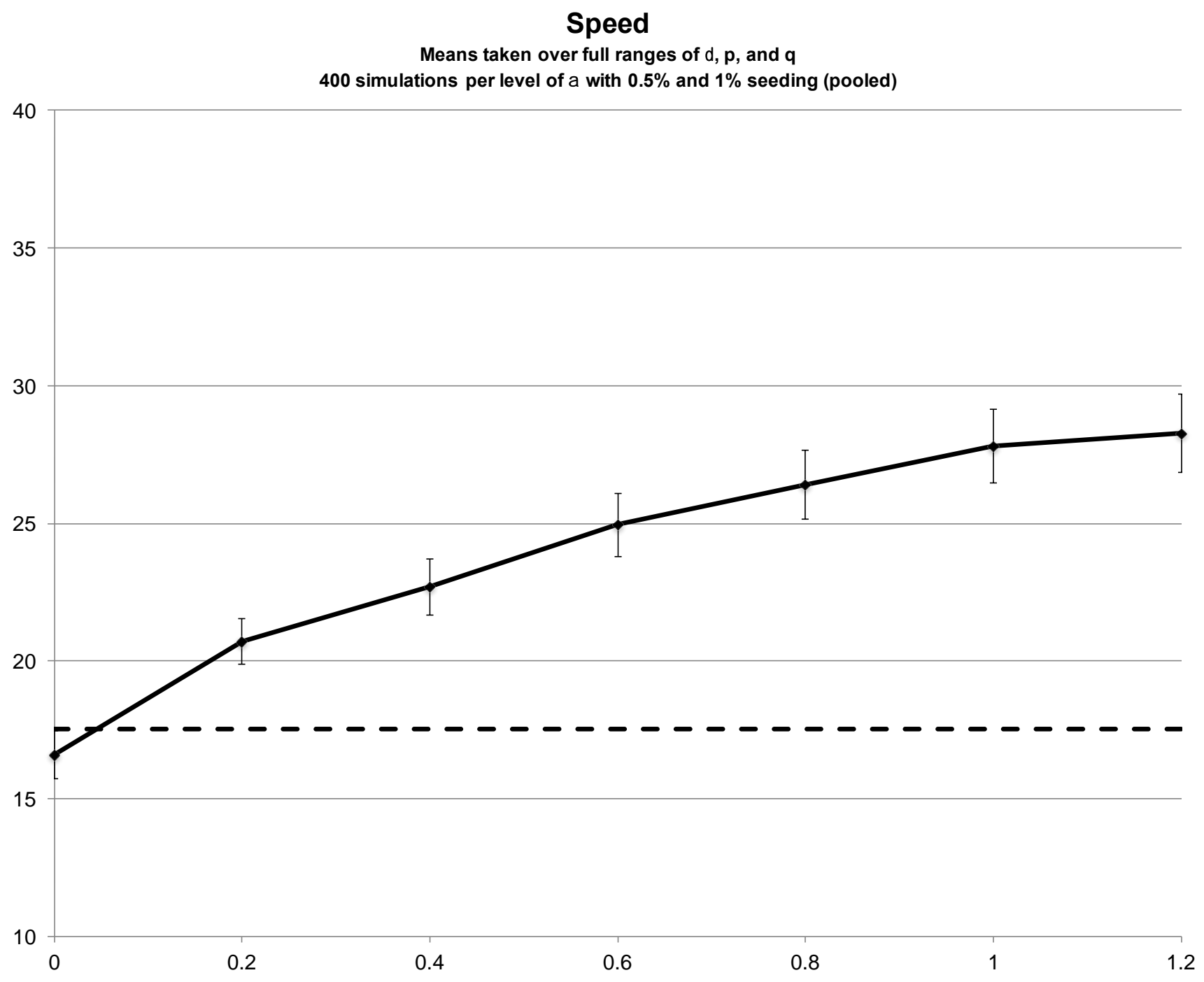




\section{Web Appendix A: Preliminary Surveys/Assumption Tests}

\section{Do Transmitters Consider Receiver Connectivity?}

We assume that an incentive or, more generally, some kind of coordination mechanism, is needed for getting customers to transmit WOM to higher-connectivity friends because they usually do not think about receiver (friend) connectivity when deciding with whom they will share information. This assumption is confirmed in the first survey; i.e., we show that receiver connectivity is not typically considered when people transmit WOM. Four hundred and forty-eight members of a large U.S. online panel were surveyed. Participants were asked to think of themselves in a situation where they were transmitting information about a product/service to someone they knew (e.g., friend, coworker, relative) and, for this situation, to write down the most important receiver characteristic that came to mind. ${ }^{6}$ We categorized the important receiver characteristics that participants provided into categorized into three categories: (i) listening (e.g., "I want a receiver who will listen to me"), (ii) connectivity (e.g., "I want a receiver who is well connected"), and (iii) other. Consistent with our assumption, only 17 out of 448 participants (3.8\%) listed a characteristic that was related to either a receiver's connectivity or ability to disseminate information. Conversely, 256 participants (57.1\%) listed a receiver's willingness to listen, with 114 participants specifically using the phrase "good listener." The remaining 175 (39.1\%) participants fell into several other categories, including wanting the receiver to be intelligent, interesting, or a close friend. These results suggest that transmitters are unlikely to place much weight on connectivity when deciding to whom to transmit WOM.

\section{Web Appendix B: Stimuli/Manipulations Used in Experiments}

\section{B1. Study 1}

Thank you for completing the previous task and survey. Before you continue to the next part of today's lab session we'd like to tell you about a new marketing research lab that we plan to open in late 2013 and get your help with something. This will only take a few minutes.

At present, the only way for you to participate in research studies like those you are doing today is on select Fridays during the Fall and Spring semesters. As you know, you receive course credit for this. We will be opening a new lab facility on campus that will run during the week. You will be able to sign up for studies online and you will be PAID for your participation. The facility will be located on campus.

If you are interested in being informed over email about this new paid lab sessions opportunity please enter your email address in the box below. Your email address will only be used to inform you about this lab and will not be shared with anyone other than the lab management team.

\section{[Control Condition]}

Finally, note that once this new lab gets started we will need to spread the word about it. Once this starts up later in the year we need it to get off the ground quickly, and that means having plenty of students aware of it and willing to sign up for paid lab sessions. We will rely heavily on word of mouth to get the news out to the university community.

\footnotetext{
${ }^{6}$ Participants also rated situation familiarity $(1=$ extremely unfamiliar to $7=$ extremely familiar; $\mathrm{M}=5.66, \mathrm{SD}=1.12)$.
} 
At this stage we are asking for a little help from you. Please suggest the name of just ONE STUDENT (not you!) who you think would like to hear about this and could help spread the word. Enter their name in the box below. We will look up their university email address and put them on our contact list.

\section{[Positive Interdependence Condition]}

Finally, note that once this new lab gets started we will need to spread the word about it. If not enough students around the university sign up for sessions those sessions will be cancelled. That will mean that students - perhaps you - who do want to participate and earn some extra money won't be able to unless we have a "critical mass" of students who are willing to participate. Once this starts up later in the year we need it to get off the ground quickly, and that means having plenty of students aware of it and willing to sign up for paid lab sessions. We will rely heavily on word of mouth to get the news out to the university community.

At this stage we are asking for a little help from you. Please suggest the name of just ONE STUDENT (not you!) who you think would like to hear about this and could help spread the word. Enter their name in the box below. We will look up their university email address and put them on our contact list.

\section{B2. Study 2}

[Control condition, Urgent condition]

Imagine the following scenario:

You received the following promotional email from Amazon.com. Please read the details very carefully and make sure the promotion is clear to you.

\section{$\underline{\text { Amazon.com Flash Sale on Gift Cards }}$}

You have been selected to receive a special offer from Amazon.com, but hurry because this is a limited-time offer!

Tomorrow, Tuesday, starting at 12:01 am (US PT) and ending at 11:59pm (US PT) you can save 50\% on the purchase of an Amazon gift card with a face value up to \$250. This means that, for example, if you wanted to buy an Amazon gift card worth $\$ 100$, during this Flash Sale you would only pay \$50. This offer is valid for all types of Amazon gift cards (electronic, print-at-home, send-by-mail) purchased through Amazon.com/Giftcards using the discount code FLASH50 when you checkout. (Limit one per billing address.)

Is there a catch? No! You and anyone else with this code can receive the discount by making a gift card purchase using this code during the 24 hour Flash Sale period tomorrow.

\section{[Externality condition, Urgent condition]}

Imagine the following scenario: 
You received the following promotional email from Amazon.com. Please read the details very carefully and make sure the promotion is clear to you.

\section{$\underline{\text { Amazon.com Flash Sale on Gift Cards }}$}

You have been selected to receive a special offer from Amazon.com, but hurry because this is a limited-time offer!

Tomorrow, Tuesday, starting at 12:01 am (US PT) and ending at 11:59pm (US PT) you can save 50\% on the purchase of an Amazon gift card with a face value up to \$250. This means that, for example, if you wanted to buy an Amazon gift card worth $\$ 100$, during this Flash Sale you would only pay $\$ 50$. This offer is valid for all types of Amazon gift cards (electronic, print-at-home, send-by-mail) purchased through Amazon.com/Giftcards using the discount code FLASH50 when you checkout. (Limit one per billing address.)

Is there a catch? Yes! For you and anyone else with this code to receive the discount, a minimum of 50,000 people must make a gift card purchase using this code during the 24 hour Flash Sale period tomorrow. If this minimum number of purchases is not reached then you will not receive the discounted gift card and will not be charged.

\section{[Control condition, Not urgent condition]}

Imagine the following scenario:

You received the following promotional email from Amazon.com. Please read the details very carefully and make sure the promotion is clear to you.

\section{$\underline{\text { Amazon.com Flash Sale on Gift Cards }}$}

You have been selected to receive a special offer from Amazon.com, but hurry because this is a limited-time offer!

Tomorrow, Tuesday, starting at 12:01am (US PT) and ending next Monday at 11:59pm (US PT) you can save $50 \%$ on the purchase of an Amazon gift card with a face value up to $\$ 250$. This means that, for example, if you wanted to buy an Amazon gift card worth \$100, during this Flash Sale you would only pay $\$ 50$. This offer is valid for all types of Amazon gift cards (electronic, print-at-home, send-bymail) purchased through Amazon.com/Giftcards using the discount code FLASH50 when you checkout. (Limit one per billing address.)

Is there a catch? No! You and anyone else with this code can receive the discount by making a gift card purchase using this code during the 7 day Flash Sale period starting tomorrow.

\section{[Externality condition, Not urgent condition]}

Imagine the following scenario:

You received the following promotional email from Amazon.com. Please read the details very carefully and make sure the promotion is clear to you. 
$\underline{\text { Amazon.com Flash Sale on Gift Cards }}$

You have been selected to receive a special offer from Amazon.com, but hurry because this is a limited-time offer!

Tomorrow, Tuesday, starting at 12:01am (US PT) and ending next Monday at 11:59pm (US PT) you can save $50 \%$ on the purchase of an Amazon gift card with a face value up to $\$ 250$. This means that, for example, if you wanted to buy an Amazon gift card worth $\$ 100$, during this Flash Sale you would only pay $\$ 50$. This offer is valid for all types of Amazon gift cards (electronic, print-at-home, send-bymail) purchased through Amazon.com/Giftcards using the discount code FLASH50 when you checkout. (Limit one per billing address.)

Is there a catch? Yes! For you and anyone else with this code to receive the discount, a minimum of 50,000 people must make a gift card purchase using this code during the 7 day Flash Sale period starting tomorrow. If this minimum number of purchases is not reached then you will not receive the discounted gift card and will not be charged.

\section{B3. Study 3}

\section{[Control condition]}

This Year Spread The Word About The Flu, Not The Flu

The 2014-2015 flu season is almost upon us here in the United States, and many indicators point to it being potentially more severe than recent past flu seasons. Right now, in September, there are relatively low levels of flu throughout most of the US. This is expected to change rapidly as temperatures drop and winter approaches.

The influenza vaccine (or "flu shot") is now widely available throughout the United States at drug stores, health clinics. The CDC's guidelines note that there are different types of flu shots approved for people of different ages, but there are flu shots approved for use in people as young as 6 months of age and up. Flu shots are also approved for use in pregnant women and people with chronic health conditions. It is recommended that everyone 6 months of age and older get the flu shot this year, ideally before October. (Please note: before getting a flu shot you should always consult with a health care professional, such as a physician, nurse, or pharmacist.)

It is recommended that people get their flu shot as early as possible - before the beginning of October is best.

Studies have shown that the earlier more people get their flu shot, the lower the chance of you getting the flu. Getting the flu shot before October also has been shown to significantly reduce the chances of the flu spreading quickly among the population and becoming a serious pandemic.

This year, the CDC reminds Americans that it is everyone's responsibility to help reduce the spread of the flu. You can help by spreading the word about the flu - remind people to get their flu shot now. 
Economists estimate that the flu costs the U.S. economy upwards of $\$ 10$ billion each year. If there is a flu pandemic, that cost rises to $\$ 100$ billion. Each year the government spends your tax dollars on covering the direct and indirect costs of the flu.

Fortunately, this cost can be drastically reduced if more people get their annual flu shot early in the season, ideally before the beginning of October. So, this year get your flu shot now and help us spread the word to others.

The more people who get their flu shot before October, the better off YOU will be. This year spread the word about the flu, not the flu.

Here's how YOU can help today

The U.S. government has a website Flu.gov that is an easy to understand source of information about the flu and flu shot options available to people. We would like you to email a link to this site to a friend, as a way of doing your part to spread the word about the flu, not the flu.

Take a moment to think about who you want to email, and then click the email icon below. Clicking this icon will open a new browser tab or window. Follow the steps on there for emailing a link to Flu.gov's flu shot page to your friend. They can read about the benefits of getting a flu shot, and even search for places in their zip code that administer flu shots.

Privacy notice: this icon opens up a new browser tab or window in which you have to enter email addresses (your friend's and your own). An email identified as coming from you will then be sent to your friend. A link to Flu.gov will be in that email. The email addresses you enter will be 100\% private. They are not stored and will not be known by anyone. Your information is safe. A third-party email-based link-sharing service is used for this and the requester of this HIT will NOT know the email addresses you enter.

Click this icon to open up the interface for emailing a link to Flu.gov to your friend: [email icon]

\section{[Externality condition]}

This Year Spread The Word About The Flu, Not The Flu

The 2014-2015 flu season is almost upon us here in the United States, and many indicators point to it being potentially more severe than recent past flu seasons. Right now, in September, there are relatively low levels of flu throughout most of the US. This is expected to change rapidly as temperatures drop and winter approaches.

The influenza vaccine (or "flu shot") is now widely available throughout the United States at drug stores, health clinics. The CDC's guidelines note that there are different types of flu shots approved for people of different ages, but there are flu shots approved for use in people as young as 6 months of age and up. Flu shots are also approved for use in pregnant women and people with chronic health conditions. It is recommended that everyone 6 months of age and older get the flu shot this year, ideally before October. (Please note: before getting a flu shot you should always consult with a health care professional, such as a physician, nurse, or pharmacist.) 
It is recommended that people get their flu shot as early as possible - before the beginning of October is best.

Studies have shown that the earlier more people get their flu shot, the lower the chance of you getting the flu. Getting the flu shot before October also has been shown to significantly reduce the chances of the flu spreading quickly among the population and becoming a serious pandemic.

This year, the CDC reminds Americans that it is everyone's responsibility to help reduce the spread of the flu. You can help by spreading the word about the flu - remind people to get their flu shot now.

Economists estimate that the flu costs the U.S. economy upwards of $\$ 10$ billion each year. If there is a flu pandemic, that cost rises to $\$ 100$ billion. Each year the government spends your tax dollars on covering the direct and indirect costs of the flu.

Fortunately, this cost can be drastically reduced if more people get their annual flu shot early in the season, ideally before the beginning of October. So, this year get your flu shot now and help us spread the word to others.

The more people who get their flu shot before October, the better off YOU will be. This year spread the word about the flu, not the flu.

Here's how YOU can help today

The U.S. government has a website Flu.gov that is an easy to understand source of information about the flu and flu shot options available to people. We would like you to email a link to this site to a friend, as a way of doing your part to spread the word about the flu, not the flu.

Take a moment to think about who you want to email, and then click the email icon below. Clicking this icon will open a new browser tab or window. Follow the steps on there for emailing a link to Flu.gov's flu shot page to your friend. They can read about the benefits of getting a flu shot, and even search for places in their zip code that administer flu shots.

As a way of thanking you for doing your part to spread the word about the flu, not the flu, by sending this email to a friend you will have a chance to receive a $\$ 2$ bonus payment for this HIT.

Here's how it works - the link that will automatically be generated in the email to your friend is unique to you. Our software tracks this link and tells us how many people visit Flu.gov because of you sharing this link with your friend today. If 50 or more people visit Flu.gov through your unique link by 12 noon Eastern Time tomorrow [date] we will pay you a $\$ 2$ bonus. It is that simple.

Privacy notice: this icon opens up a new browser tab or window in which you have to enter email addresses (your friend's and your own). An email identified as coming from you will then be sent to your friend. A link to Flu.gov will be in that email. The email addresses you enter will be 100\% private. They are not stored and will not be known by anyone. Your information is safe. A third-party email-based link-sharing service is used for this and the requester of this HIT will NOT know the email addresses you enter.

Click this icon to open up the interface for emailing a link to Flu.gov to your friend: [email icon] 